\title{
The Potential of Genetically Engineered Magnetic Particles in Biomedical Applications
}

\author{
Tomoko Yoshino, Yuka Kanetsuki and Tadashi Matsunaga \\ Tokyo University of Agriculture and Technology \\ Japan
}

\section{Introduction}

Magnetic particles are currently one of the most important materials in the industrial sector, where they have been widely used for biotechnological and biomedical applications such as carriers for recovery and for detection of DNA, proteins, viruses, and cells (Perez et al., 2002; Kramer et al., 2004; Gonzales and Krishnan, 2005). The major advantage of magnetic particles is that they can be easily manipulated by magnetic force, which enables rapid and easy separation of target molecules bound to the particles from reaction mixtures (Mirzabekov et al., 2000; Gu et al., 2003; Kuhara et al., 2004; Xu et al., 2004). Use of magnetic particles is beneficial for complete automation of steps, resulting in minimal manual labor and providing more precise results (Sawakami-Kobayashi et al., 2003). Biomolecules such as DNA, biotin, and antibodies have been assembled onto magnetic particles and used as recognition materials for target recovery, separation, or detection.

The method chosen for biomolecule assembly is determined by the surface properties of the magnetic particles. Various methods of assembly onto magnetic particles have been reported such as electrostatic assembly (Goldman et al., 2002), covalent cross-linking (Grubisha et al., 2003; Gao et al., 2004) avidin-biotin technology (Gref et al., 2003), membrane integration (Mirzabekov et al., 2000; Tanaka et al., 2004), and gene fusion techniques (Nakamura et al., 1995b; Yoshino et al., 2004; Yoshino and Matsunaga, 2006). The amount and stability of assembled biomolecules and the percentage of active biomolecules among assembled molecules are dependent on the method used for coupling. However, the fabrication techniques have not been standardized. As applications for magnetic particles in the biotechnology field increase, magnetic particles with greater functionality and novel methods for their production are in demand.

Magnetotactic bacteria synthesize uniform, nano-sized magnetite $\left(\mathrm{Fe}_{3} \mathrm{O}_{4}\right)$ particles, which are referred to as "bacterial magnetic particles" (BacMPs). A thin lipid bilayer membrane envelops the individual BacMP, which confers high and even dispersion in aqueous solutions as compared to artificial magnetic particles, making them ideal biotechnological materials (Matsunaga et al., 2003). To use these particles for biotechnological applications, it is important to attach functional molecules such as proteins, antibodies, peptides, or DNA. BacMP-specific proteins have been used as anchor proteins, which facilitate efficient localization and appropriate orientation of various functional proteins attached to BacMPs. We have developed several methods for modification and assembly of these functional organic molecules over the surface of BacMPs using chemical and genetic techniques. In this chapter, we describe advanced magnetic particles used in biomedical applications and the 
methods for bioengineering of these particles. Specific focus is given to the creation of functional BacMPs by magnetotactic bacteria and their applications.

\section{Production of functional magnetic particles}

Currently, magnetic particles offer vast potential for ushering in new techniques, especially in biomedical applications, as they can be easily manipulated by magnetic force. The important characteristics of these particles include (1) immobilization of higher numbers of probes onto magnetic particles because particle surfaces are wider than those of a flat surface, (2) reduction of reaction times because of good dispersion properties that increase reaction efficiency, (3) facilitation of the bound/free separation step with a magnet, without centrifugation or filtration, and (4) the use of automated robotic systems for all reaction steps. These characteristics offer great benefits for biomedical applications such as rapid and precise measurements or separations of bio-targets. Here, the methods for production of functional magnetic particles are introduced.

\subsection{Commercialized magnetic particles}

Commercialized magnetic particles are usually composed of superparamagnetic iron oxide nanoparticles $\left(\mathrm{Fe}_{3} \mathrm{O}_{4}\right.$ or $\left.\mathrm{Fe}_{2} \mathrm{O}_{3}\right)$, which exhibit magnetic properties only in the presence of external magnetic fields. These particles are embedded in polymers such as polysaccharides, polystyrene, silica, or agarose. Micro-sized magnetic particles can be easily removed from suspension with magnets and easily suspended into homogeneous mixtures in the absence of an external magnetic field (Ugelstad et al., 1988). Furthermore, functional groups or biomolecules for the recognition of targets are conjugated to the polymer surfaces of magnetic particles (Fig. 1), and targets can be collected, separated, or detected by the magnetic particles.

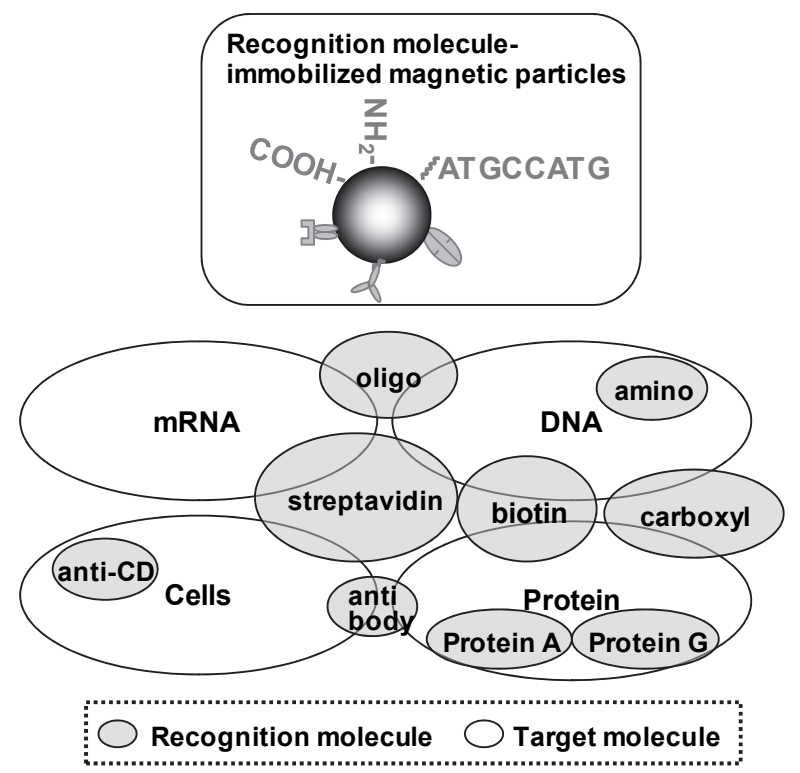

Fig. 1. Use of general magnetic particles 
Biotin or streptavidin-assembled magnetic particles, on which complementary nucleic acid strands are immobilized, are widely used for the recovery or extraction of specific nucleic acids and are marketed worldwide. Moreover, magnetic particles can be used as supports for separation or detection of proteins or cells. For example, protein A- or protein G-assembled magnetic particles are suitable for antibody purification and are more efficient than columnpurification techniques.

Currently, polymer magnetic particles marketed as Dynabeads ${ }^{\circledR}$ (Invitrogen, co.) are one of the most widely used magnetic particles for biotechnology applications (Sawakami-Kobayashi et al., 2003; Prasad et al., 2003). These particles are prepared from mono-sized macroporous polystyrene particles that are magnetized by an in situ formation of ferromagnetic materials inside the pores. Dynabeads ${ }^{\circledR}$ with diameters of $2.8 \mu \mathrm{m}$ or $4.5 \mu \mathrm{m}$ are the most widely used magnetic particles by scientists around the world, particularly in the fields of immunology, cellular biology, molecular biology, HLA diagnostics, and microbiology.

Antibody-immobilized magnetic particles have been used preferentially in target-cell separation of leukocytes (Stampfli et al., 1994; Schratzberger et al., 1997; Schwalbe et al., 2006; Nakamura et al., 2001) for in vitro diagnosis because of the simpler and more rapid methodology as compared to cell sorting using a flow cytometer. These commercially available magnetic particles are chemically synthesized compounds of micrometer and nanometer sizes. Several cell separation systems using nano-sized magnetic particles, such as 50-nm iron oxide particles with polysaccharide- (Miltenyi Biotech, co.) or dextran- (StemCell Technologies Inc.) coated superparamagnetic nanoparticles, are commercially available (Miltenyi, 1995; Wright, 1952). Because these particles are superparamagnetic and are preferred for high-gradient magnetic separation, specially-designed magnetic columns that produce high magnetic field gradients are required for cell separation (Miltenyi, 1995). Nanosized magnetic particles are advantageous for assay sensitivity, rapidity, and precision. However, it remains difficult to synthesize nano-sized magnetic particles with uniform size and shape that adequately disperse in aqueous solutions. Consequently, advanced techniques and high costs are required for the production of nano-sized magnetic particles.

Magnetic particles are widely used not only as carriers for recovery or detections of biomolecules, but also used as probes for magnetic detections, or agent for magnetic-fieldinduced heating. Especially, magnetic particles that have high saturation magnetization are ideal candidates for MRI contrast agents, and various kinds of magnetic particles have been developed and used for diagnoses. Recently, Mulder et. al. developed the paramagnetic quantum dots (pQDs) coated with paramagnetic and pegylated lipids which had a high relaxivity. The high relaxivity makes the pQDs contrast agent an attractive candidate for molecular MRI purposes. This nanoparticulate probe makes it detectable by both MRI and fluorescence microscopy (Mulder et al., 2006). It was successful that the synthesis of quantum dots with a water-soluble and paramagnetic micellular coating were used as a molecular imaging probe for both fluorescence microscopy and MRI. The present study uses magnetic nanoparticles as bimodal tools and combines magnetically induced cell labelling and magnetic heating. The particles are used in hyperthermia agents, where the magnetic particles are heated selectively by application of an high frequency magnetic field (Mulder et al., 2006). These magnetic heating treatments using superparamagnetic iron oxide nanoparticles continue to be an active area of cancer research. The research aimed to assess if a selective and higher magnetic nanoparticles accumulation within tumor cells is due to magnetic labeling and consequently a larger heating effect occurs after exposure to an alternating magnetic field in order to eliminate labeled tumor cells effectively (Kettering et 
al., 2007). Moreover, in recent years magnetic devised like giant magnetoresistive (GMR) sensors have shown a great potential as sensing elements for biomolecule detection (Baselt et al., 1998; Edelstein et al., 2000; Schotter et al., 2004). The GMR biochip based on spin valve sensor array and magnetic nanoparticle probes was developed for inexpensive, sensitive and reliable DNA detection using plasmid-derived samples ( $\mathrm{Xu}$ et al., 2008). The applications of magnetic particles as probes are increasingly advanced in biomolecule quantitative analysis.

\subsection{Magnetic particles produced by magnetotactic bacteria}

Magnetotactic bacteria synthesize nano-sized biomagnetites, otherwise known as bacterial magnetic particles (BacMPs), that are enveloped individually by a lipid bilayer membrane (Blakemore, 1983). BacMPs are ultrafine magnetite crystals (50-100 nm in diameter) with uniform morphology produced by Magnetospirillum magneticum AMB-1 (Fig. 2).

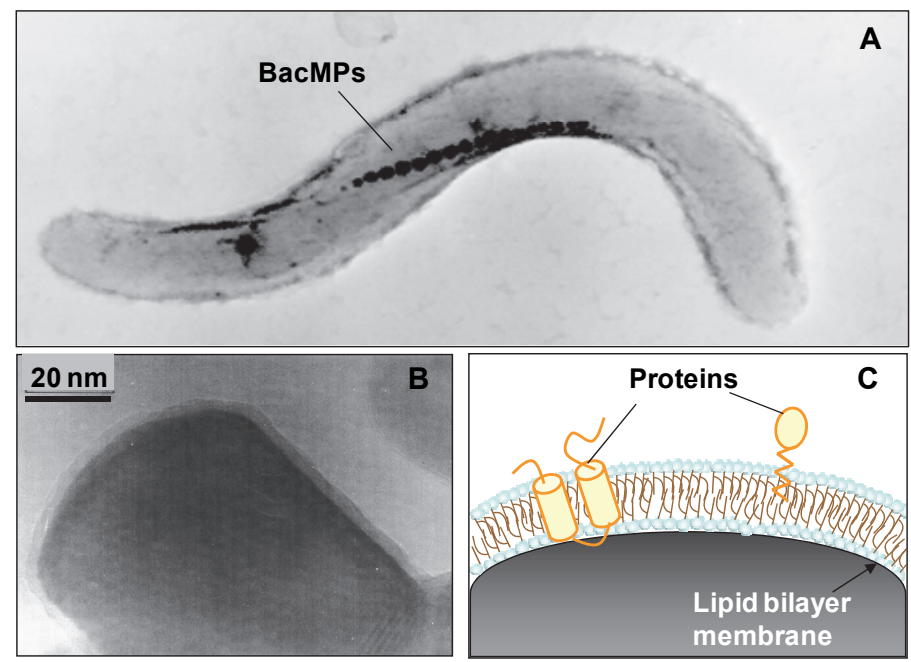

Fig. 2. Transmission electron microscopic (TEM) image and schematic diagram of Magnetospirillum magneticum AMB-1 (A), bacterial magnetic particles (BacMPs, B) and schematic diagram of proteins on the BacMPs surface $(C)$.

The molecular mechanism of BacMP synthesis involves a multiple-step process that includes vesicle formation, iron transport, and magnetite crystallization. This mechanism has been studied using genomic, proteomic, and bioinformatic approaches (Matsunaga et al., 2005; Nakamura et al., 1995a; Arakaki et al., 2003; Amemiya et al., 2007) , and a comprehensive analysis provided a clear view of the elaborate regulation of BacMP synthesis.

Techniques for the mass cultivation of magnetotactic bacteria have been developed, allowing for a steady supply of BacMPs for industrial applications. Based on the molecular mechanism of BacMP formation in $M$. magneticum AMB-1, designed functional nanomaterials have also been developed. Through genetic engineering, functional proteins such as enzymes, antibodies, and receptors have been displayed on the surface of BacMPs. The display of proteins on BacMPs was achieved using a fusion technique involving anchor proteins isolated from magnetotactic bacteria (Nakamura et al., 1995b). Figure 3A shows the 
procedure for producing functional magnetic particles through genetic engineering of these bacteria. Several proteins involved in the magnetic biosynthetic mechanism are embedded in the BacMP membrane. In M. magneticum AMB-1, MagA (46.8 kDa), Mms16 (16 kDa), and Mms13 (13 kDa) proteins have been used as anchor molecules for displaying functional proteins (Nakamura et al., 1995b; Yoshino et al., 2004; Matsunaga et al., 2005; Matsunaga et al., 1999; Matsunaga et al., 2000).

A

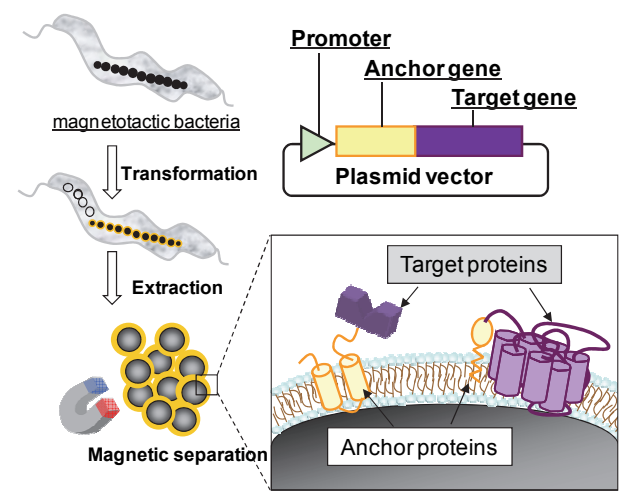

B

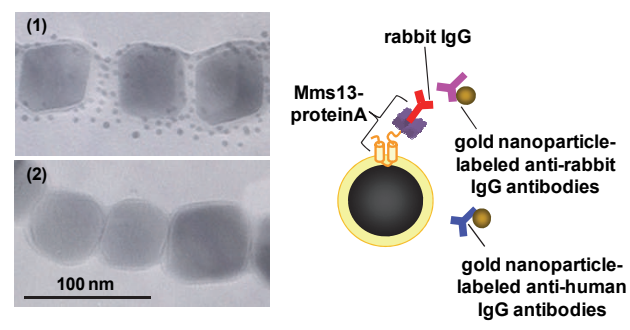

Fig. 3. Preparation of BacMPs displaying functional proteins.

(A) The functional protein gene is fused to an anchor gene for display of a functional protein on BacMPs. A plasmid harboring the fusion gene is introduced into M. Magneticum AMB-1.

(B) TEMs of BacMPs displaying protein A which were treated with rabbit IgG after addition of gold nanoparticle (5 nm)-labeled anti-rabbit IgG antibodies (1) or anti-human IgG antibodies (2).

MagA was one of the first proteins experimentally demonstrated to be localized on the surface of BacMPs (Nakamura et al., 1995a; Nakamura et al., 1993). MagA is a transmembrane protein identified from a M. magneticum AMB-1 mutant strain generated by transposon mutagenesis (Nakamura et al., 1995a). As proof of localization, luciferase (61 $\mathrm{kDa}$ ) was fused to the C-terminus of MagA (Nakamura et al., 1995b). This was the first report of protein display on BacMPs using gene fusion techniques. However, the efficiency and stability of proteins displayed on BacMPs were limited, and only a few molecules were displayed on a single BacMP.

As research in this field progressed, a more effective and stable method for protein display was developed. To establish high levels of expressed proteins displayed on BacMPs, strong promoters and stable anchor proteins were identified using M. magneticum AMB-1 genome and proteome analysis (Yoshino and Matsunaga, 2005).

An integral BacMP membrane protein, Mms13, was isolated as a stable anchor molecule, and its anchoring properties were confirmed by luciferase fusion studies. The C-terminus of Mms13 was expressed on the surface of BacMPs, and Mms13 was tightly bound to the magnetite directly, permitting stable localization of luciferase on BacMPs. Consequently, the luminescence intensity obtained from BacMPs using Mms13 as an anchor molecule was more than 1,000-times greater than when MagA was used. Furthermore, the IgG-binding domain of protein A was displayed uniformly on BacMPs using Mms13 (Fig. 3B). 
Strong promoters and stable anchor proteins allowed efficient display of functional proteins on BacMPs. However, the display of particular proteins remained a technical challenge due to the cytotoxic effects of the proteins when they were overexpressed in bacterial cells. Specifically, transmembrane proteins such as G-protein coupled receptors were still difficult to express in magnetotactic bacteria. An inducible protein expression system is often used to control the expression dose and timing of transmembrane proteins. Recently, we developed a tetracycline-inducible protein expression system in M. magneticum AMB-1 to prevent the toxic effects of transmembrane protein expression (Yoshino et al., 2010). This system was implemented to obtain the expression of tetraspanin CD81, where the truncated form of CD81, including the ligand binding site, was successfully expressed on the surface of BacMPs using Mms13 as an anchor protein and the tetracycline-inducible protein expression system. These results suggest that the inducible expression system will be a useful tool for the expression and display of transmembrane and other potentially cytotoxic proteins on the membranes of BacMPs.

Currently, many types of functional proteins can be displayed at high levels on magnetic particles due to the modifications described above. Generally, immobilization of proteins onto magnetic particles is performed by chemical cross-linking; however, this can hinder the activity of some proteins. Because the amine-reactive cross-linker can bind to proteins in a random manner, the target proteins may become inactivated. Furthermore, protein orientation on the solid phase is difficult to control during chemical conjugation. To overcome these difficulties, protein display on magnetic particles produced by magnetotactic bacteria through gene fusion is a promising approach, and the techniques have expanded the number of applications.

\section{Applications of magnetic particles}

Magnetic iron oxide particles, such as magnetite $\left(\mathrm{Fe}_{3} \mathrm{O}_{4}\right)$ and maghemite $\left(\gamma-\mathrm{Fe}_{2} \mathrm{O}_{3}\right)$, are widely used in medical and diagnostic applications such as magnetic resonance imaging (Gleich and Weizenecker, 2005), cell separation (Miltenyi et al., 1990) , drug delivery (Plank et al., 2003), and hyperthermia (Pardoe et al., 2003). To use these particles for biotechnological applications, the surface modification of the magnetic particle with functional molecules such as proteins, peptides, or DNA must be considered. Previously, only DNA- or antibody-immobilized magnetic particles were marketed and used in biotechnology; it was suggested that the techniques for the immobilization of enzymes or receptors were more complicated and time consuming. However, as the methods for assembling functional proteins onto magnetic particles have become simpler and more efficient, the applications of magnetic particles have expanded. Here, the applications of BacMPs displaying functional proteins such as antibody, enzyme, or receptor are described.

\subsection{Applications of antibody-magnetic particles}

Magnetic particles have been widely used as carriers of antibodies for immunoassay, cell separation, and tissue typing (Herr et al., 2006; Tiwari et al., 2003; Weissleder et al., 2005). The use of magnetic particles is advantageous for full automation, minimizing manual labor and providing more precise results (Sawakami-Kobayashi et al., 2003; Tanaka and Matsunaga, 2000). In particular, immunomagnetic particles have been used preferentially in target cell separation from leukocytes (Stampfli et al., 1994; Schratzberger et al., 1997) for in vitro diagnosis, as this provides a more rapid and simple methodology compared with cell sorting using a flow cytometer. 
To immobilize antibody, protein A, which is the antibody-binding protein derived from Staphylococcus aureus (Deisenhofer, 1981), has been immobilized on magnetic particles using the sequence of the Z-domain, a synthetic analogue of the IgG-binding B-domain. Staphylococcus protein A consists of a cell wall binding region and five domains, termed C, B, $\mathrm{A}, \mathrm{D}$, and $\mathrm{E}$, with $\mathrm{C}$ next to the cell wall. The molecular interaction of protein A with IgG is well understood, and the binding sites on the Fc domain of IgG1, -2 , and -4 have been characterized. X-ray analysis has revealed that the B-domain of protein A has two contact sites that interact with the Fc domain of IgG (Eliasson and Kogelschatz, 1988). Based on this knowledge, a synthetic Z-domain, which consists of 58 amino acids and is capable of binding the Fc domain, has been constructed (Lowenadler et al., 1987) . IgG can bind the Zdomain on magnetic particles with uniform orientation.

Z-domain was displayed on bacterial magnetic particles using gene fusion techniques and was used to detect human insulin from whole blood by sandwich enzyme immunoassays. The experimental procedure was fully automated using a pipetting robot bearing a magnet (Tanaka and Matsunaga, 2000).

Antibody-conjugated BacMPs also can be utilized for cell separation. In general, nano-sized magnetic particles, rather than micro-sized particles, are preferred for cell separation because separated cells with nano-sized magnetic particles on their surfaces can be used in subsequent flow cytometric analysis (Graepler et al., 1998). Additionally, micro-sized magnetic particles are more likely to have inhibitory effects on cell growth and differentiation after magnetic separation.

Magnetic separation permits target cells to be isolated directly from crude samples such as blood, bone marrow, tissue homogenates, or cultivation media. Compared to other more conventional methods of cell separation, magnetic separation may be considered a sample enrichment step for further chromatographic and electromigratory analysis. To enrich for target cells, cell surface antigens, such as cluster of differentiation (CD) antigens, were used as markers. CD8, CD14, CD19, CD20, and CD34 positive cells were efficiently enriched from peripheral blood (Kuhara et al., 2004; Matsunaga et al., 2006). The separated CD34 positive cells retained the capability of forming colonies as hematopoietic stem cells.

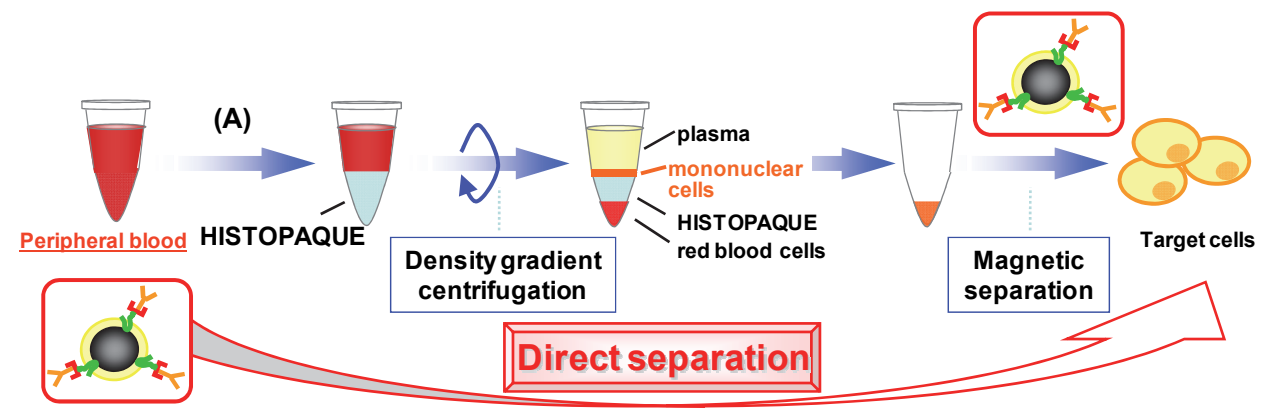

(B)

Fig. 4. Schematic illustration of cell separation procedures. (A) The initial separation of peripheral blood mononuclear cells (PBMCs) from whole blood and the subsequent magnetic separation of target cells from PBMCs using magnetic particles followed the common procedure. (B) Target cells were separated directly from whole blood using magnetic particles in the procedure for direct magnetic cell separation. 
Protein G from Streptococcus sp (Gronenborn et al., 1991) was also displayed on BacMPs, resulting in the expansion of IgG-binding diversity. Direct magnetic separation of immune cells from whole blood using protein G-BacMPs binding anti-CD monoclonal antibodies was demonstrated (Fig. 4). Using this technique, B lymphocytes (CD19+ cells) or T lymphocytes $\left(\mathrm{CD}^{+}\right.$cells) were successfully separated at a high purity.

To increase cell separation efficiency, a novel functional polypeptide, which functions to minimize nonspecific adsorption of magnetic particles to cells, was developed for surface modification of BacMPs (Takahashi et al., 2010). Previous reports had shown that the hydrophilicity or neutral charge of the particle surface was important for the reduction of nonspecific interactions between the nanoparticle and the cell surface (Fang et al., 2009; Patil et al., 2007). The designed polypeptide was composed of multiple units consisting of four asparagines $(\mathrm{N})$ and one serine $(\mathrm{S})$ residue and was referred to as the NS polypeptide. Modification of the surface of a magnetic particle with the NS polypeptide resulted in reduction of non-specific particle-particle and particle-cell interactions. NS polypeptides on magnetic nanoparticle surfaces function as a barrier to block particle aggregation and minimize nonspecific adsorption of cells to the nanoparticles; they also add the ability to recognize and bind to target cells by working as a linker to display protein $G$ on the nanoparticles (Fig. 5). When the NS polypeptide is used in a single fusion protein as a linker to display protein $\mathrm{G}$ on magnetic particles, the particle acquires the capacity to specifically bind target cells and to avoid nonspecific adsorption of non-target cells. CD19+ cells represent $4.1 \%$ of leukocytes and in peripheral blood were calculated to be less than $0.004 \%$ of the total cells. Analysis of magnetically separated cells using flow cytometry revealed that $\mathrm{CD}_{19}{ }^{+}$cells were separated directly from peripheral blood with greater than $95 \%$ purity using protein G-displaying BacMPs bound to anti-CD19 monoclonal antibodies with the NS polypeptide. Purities were approximately $82 \%$ when the NS polypeptide was not present.

A

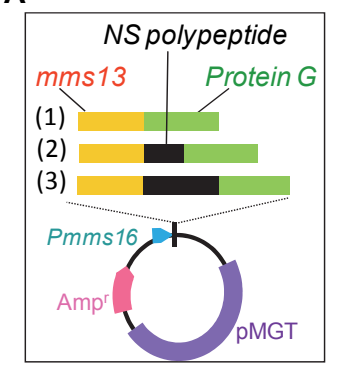

B

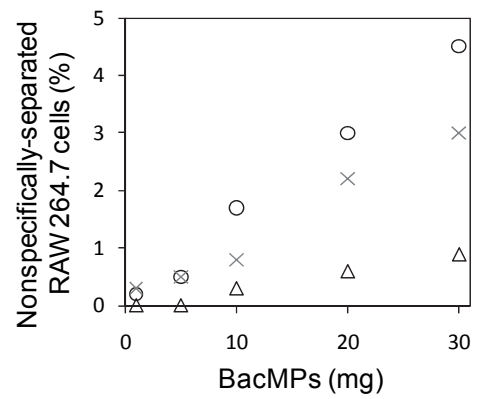

C

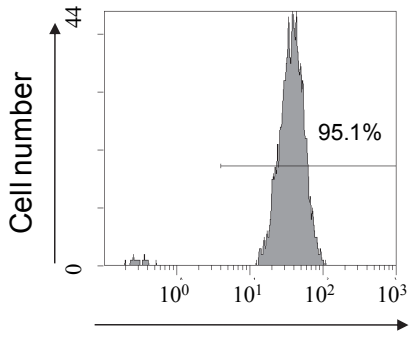

CD19

Fig. 5. Effect of NS polypeptide on cell separation.

(A) Schematic diagram of expression vectors for fusion proteins, Mms13-protein G (1), Mms13- $\left(\mathrm{N}_{4} \mathrm{~S}\right)_{10}$-protein G (2), and Mms13- $\left(\mathrm{N}_{4} \mathrm{~S}\right)_{20}$-protein G (3). (B) Correlation between the display of NS polypeptide on BacMPs and nonspecific binding of BacMPs to the cell surface. The number of RAW 264.7 cells separated using BacMPs displaying protein $G(\circ)$, BacMPs displaying $\left(\mathrm{N}_{4} \mathrm{~S}\right)_{10}$-protein $\mathrm{G}(\times)$, or BacMPs displaying $\left(\mathrm{N}_{4} \mathrm{~S}\right)_{20}$-protein $\mathrm{G}(\Delta)$ were counted, and the ratio of nonspecifically separated cells was calculated. (C) Direct magnetic separation of $\mathrm{CD} 19^{+}$cells from whole blood using BacMPs displaying $\left(\mathrm{N}_{4} \mathrm{~S}\right)_{20}$-proteins bound to PE-labeled anti-CD19 mAbs. 
Display of fusion proteins (protein G and NS polypeptide) on BacMPs significantly improved recognition of and binding to target cells, and minimized adsorption of non-target cells. These promising results demonstrated that NS polypeptides may be a powerful and valuable tool in various cell associated applications.

\subsection{Applications of enzyme-magnetic particles}

Enzymes can catalyze various biochemical reactions with high efficiency and specificity and are therefore used in industrial production (Patil et al., 2007). However, the production and purification of recombinant enzymes can be quite time and cost consuming. If enzymes could be immobilized on magnetic particles, they could be reused following magnetic recovery from the reaction mixture. Enzymes and antibodies immobilized on BacMPs using bifunctional reagents and glutaraldehyde have been found to have higher activities than those immobilized on artificial magnetic particles (Matsunaga and Kamiya, 1987). The luciferase gene (luc) was cloned downstream of the MagA promoter and the effect of iron on the regulation of MagA expression was investigated; transcription of MagA was found to be enhanced by low concentrations of iron. As an initial proof-of-concept experiment for the recovery of enzyme-displaying BacMPs, luciferase was assembled onto BacMPs (Nakamura et al., 1995b). The genes for acetate kinase and liciferase were fused to the $\mathrm{N}$ - and C-terminus of the MagA anchor protein for simultaneous display of two different enzymes (Matsunaga et al., 2000). Acetate kinase catalyzes the phosphorylation of acetate by ATP. Therefore, this reversible reaction generates ATP in the presence of ADP and acetyl phosphate. The results presented in Fig. 6 are consistent with the hypothesis that ATP is generated in situ by acetate kinase present on BacMPs through phosphorylation of ADP to ATP (Fig. 6). Thus, protein-BacMPs complexes were constructed by joining the luciferase gene to the $\mathrm{N}$ - or the C-terminal domains of MagA, and also constructed bifunctional active fusion proteins on BacMPs using MagA as an anchor with acetate kinase and luciferase.

\section{A}

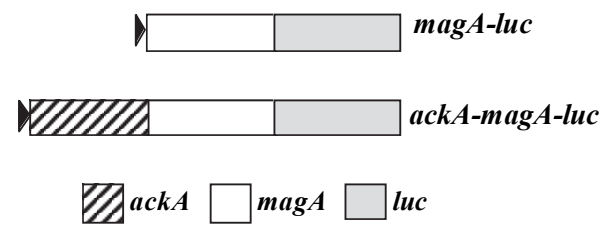

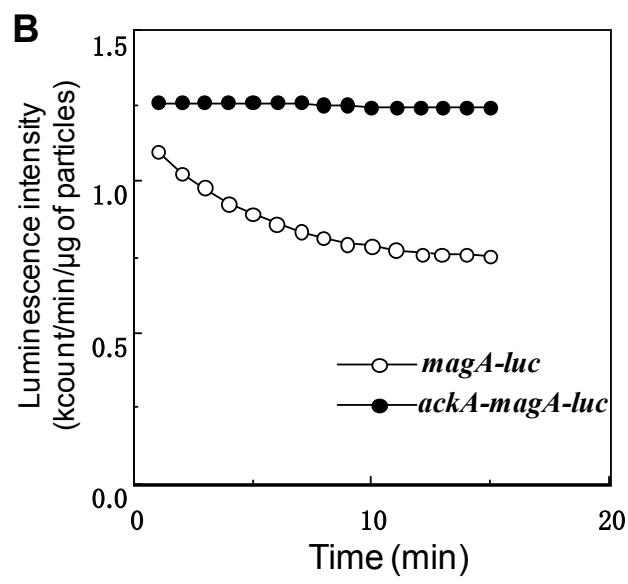

Fig. 6. Simultaneous display of two different enzymes, acetate kinase (ackA) and luciferase (luc), onto BacMPs. (A) Schematic diagram of fusion genes, and (B) luciferase activity on BacMPs 
A highly thermostable enzyme, pyruvate phosphate dikinase (PPDK), which converts pyrophosphate PPi to ATP, was also expressed on BacMPs. Pyrosequencing relies on the incorporation of nucleotides by DNA polymerase, which results in the release of PPi. The ATP produced by PPDK-displaying BacMPs can be used by luciferase in a luminescent reaction (Fig. 7). PPDK-displaying BacMPs were employed in a pyrosequencing reaction and a target oligonucleotide was successfully sequenced (Yoshino et al., 2009). The PPDK enzyme was recyclable in each sequence reaction as it was immobilized onto BacMPs which could be manipulated by a magnet. These results illustrate the advantages of using enzymedisplaying BacMPs as biocatalysts for repeat usage. Nano-sized PPDK-displaying BacMPs are useful for the scale-down of pyrosequencing reaction volumes, thus permitting highthroughput data acquisition.

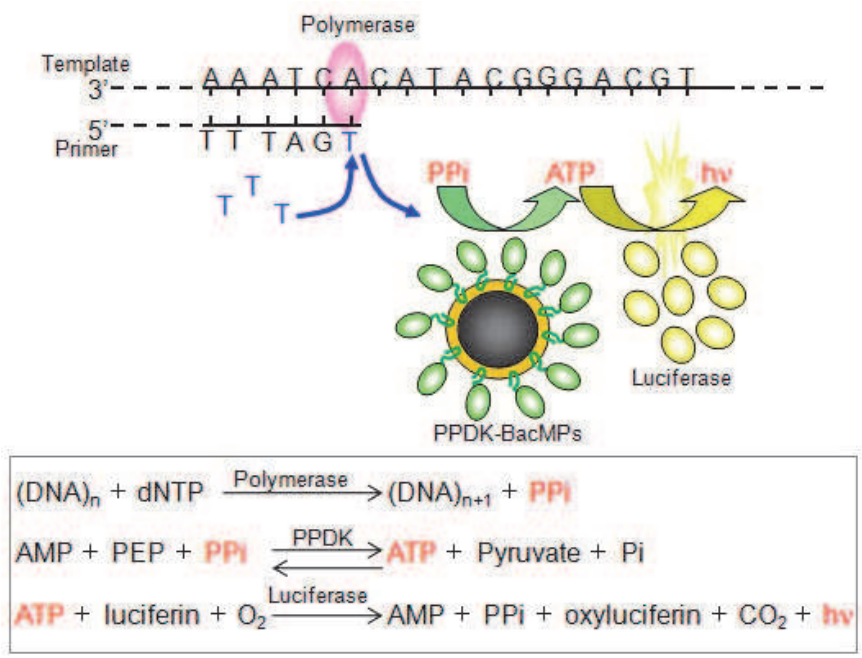

Fig. 7. Schematic diagram of the principle of pyrosequencing using PPDK-BacMPs. PEP: phosphoenolpyruvate, PPi: pyrophosphate, Pi: phosphate, PPDK: Pyruvate phosphate dikinase. PEP : phosphoenolpyruvate, PPi : pyrophosphate, Pi : phosphate, PPDK : Pyruvate phosphate dikinase

\subsection{Applications of receptor-magnetic particles}

Along with immunoassays and cell separations, ligand-binding assays to study receptor proteins are highly desired applications for magnetic particles. Receptor proteins play critical roles in gene expression, cellular metabolism, signal transduction, and intercellular communication. In particular, nuclear receptors and transmembrane receptors can be major pharmacological targets. These types of receptors have been assembled onto BacMPs.

The estrogen receptor is a nuclear receptor serving as a ligand-inducible transcriptional regulator. In recent decades, it has been suggested that natural and synthetic compounds can act as steroid hormones and adversely affect humans and wildlife through interactions with the endocrine system. These compounds have been broadly referred to as environmental endocrine disrupting chemicals (EDCs). Several chemicals, such as plastic softeners (bisphenol A) or detergents (4-nonylphenol), were originally considered harmless, 
but now are suspected of having estrogenic effects. It is probable that many unidentified chemical compounds are potential EDCs.

To evaluate and detect these chemical compounds, estrogen receptor ligand-binding domain (ERLBD) was displayed on BacMPs (Yoshino et al., 2005). ERLBD-BacMP complexes can be used for assays based on the competitive binding of alkaline phosphatase conjugated 17 $\beta$ estradiol (ALP-E2) as a tracer. The dissociation constant of the receptor was $2.3 \mathrm{nM}$. Inhibition curves were evaluated by the decrease in luminescence intensity resulting from the enzymatic reaction of alkaline phosphatase. The overall simplicity of this receptor binding assay resulted in a method that could be easily adapted to a high-throughput format.

Subsequent-generation evaluation systems for EDCs can distinguish between agonists and antagonists (Yoshino et al., 2008). In one system, ERLBD-displaying BacMPs and green fluorescent protein (GFP)-fused coactivator proteins were used in combination, and ERLBDdisplaying BacMPs were incubated with ligands and GFP-coactivators. Binding of the agonist to ERLBD induced a conformational change of ERLBD and promoted binding of the GFP-coactivator to an ERLBD dimer on the BacMP. Binding of the antagonist to ERLBD prevented the GFP-coactivator from binding to the ERLBD-BacMPs. Ligand-dependent recruitment assays of GFP-labeled coactivators to ERLBD-BacMPs were performed by measuring the fluorescence intensity (Fig. 8A). This method was used to evaluate 17ßestradiol (E2) and estriol (E3) as full agonists, octylphenol (OP) as a partial agonist, and ICI 182,780 (ICI) as an antagonist (Fig. 8B). The full agonists showed dose-dependent increases in fluorescence. Octylphenol had lower fluorescence intensity than E2, and ICI 182,780 did not produce fluorescence. The method developed in this study can be used to evaluate the estrogenic potential of chemicals by discriminating whether a chemical is an ER full agonist, a partial agonist, or an antagonist. This novel method has important potential for screening for new EDC candidates and their effects in the environment.

A

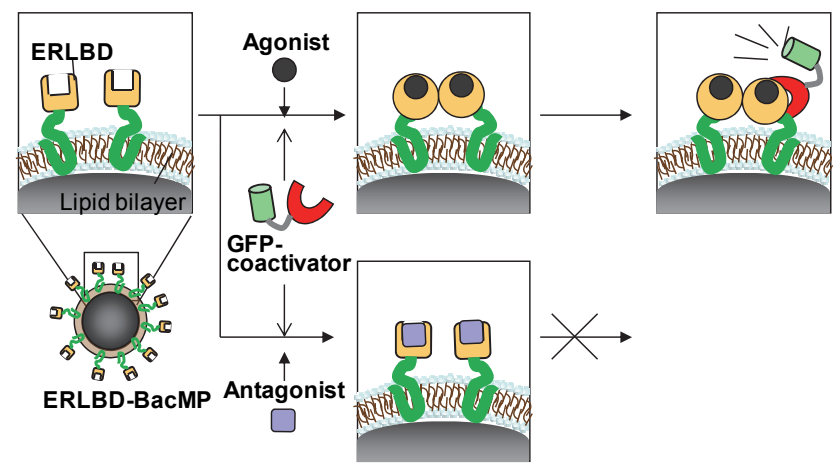

B

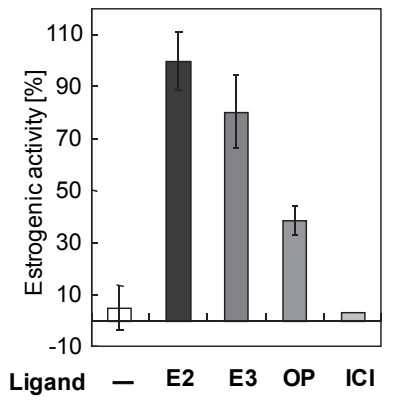

Fig. 8. Schematic diagram of the GFP-coactivator recruitment assay (A) and the assay results (B). Estrogen receptor ligand binding domain (ERLBD)-BacMPs were incubated with ligand and GFP-coactivator. Binding of agonist to ERLBD induced conformation change of ERLBD and promoted binding of GFP-coactivator to ERLBD dimmer on BacMPs. Binding of antagonist to ERLBD prevented GFP-coactivator binding to ERLBD-BacMPs. E2:17 $\beta$ Estradiol, E3:Estriol, OP:Octylphenol, ICI:ICI 182780 
G protein-coupled receptors (GPCRs) play a central role in a wide range of biological processes and are prime targets for drug discovery. GPCRs have large hydrophobic domains, and therefore, purification of GPCRs from cells is frequently time-consuming and typically results in loss of the native conformation. The D1 dopamine receptor, which is a GPCR, was successfully assembled into the lipid membrane of BacMPs (Yoshino et al., 2004). D1 dopamine receptor-displaying BacMPs were simply extracted by magnetic separation from ruptured AMB-1 transformants. This system conveniently retains the native conformation of GPCRs without the need for detergent solubilization, purification, and reconstitution after cell disruption.

Additionally, display of the tetraspanin CD81 was demonstrated using the inducible expression system (Yoshino et al., 2010) described above. CD81 is utilized when hepatitis C virus (HCV) infects hepatocytes and B lymphocytes. Therefore an inhibitor of the human CD81-HCV E2 interaction could possibly prevent HCV infection (Pileri et al., 1998). This interaction was the motivation behind efforts to produce CD81-displaying BacMPs. Consequently, the interaction between BacMPs displaying truncated CD81 and the HCV E2 envelope were detected, suggesting that CD81-displaying BacMPs could be effectively applied to identify inhibitors of the CD81-E2 interaction.

Transmembrane receptors constitute the most prominent family of validated pharmacological targets in biomedicine. Receptor-displaying BacMPs were readily extracted from ruptured AMB-1 transformants by magnetic separation, and after several washings were ready for analysis. Moreover, BacMPs are well-suited for use in a fully automated ligand-screening system that employs magnetic separation. This type of system facilitates rapid buffer exchange and stringent washing, and reduces nonspecific binding.

\section{Automated systems}

The suitability of magnetic particles for use in fully-automated systems is an important advantage in solid phases of bioassays. Automated robots bearing magnets permit rapid and precise handling of magnetic particles leading to high-throughput analysis. Different types of fully-automated systems have been developed to handle the magnetic particles and to apply them to nucleotide extraction, gene analysis, and immunoassays.

Figures 9-11 show the layout of an automated workstation with which magnetic particles are collected at the bottom of microtiter plates (Maruyama et al., 2004; Tanaka et al., 2003). For fluid handling, the processor is equipped with an automated pipetter (1) that moves in the vertical and horizontal directions. The platform contains a disposable tip rack station (2), a reagent station (3) that serves as reservoirs for wash buffers, and a reaction station (4) for a 96-well microtiter plate, where a magnetic field can be applied using a neodymium iron boron sintered (Nd-Fe-B) magnet on its underside. One pole of the $\mathrm{Nd}-\mathrm{Fe}-\mathrm{B}$ magnet applies a magnetic field to one well (Matsunaga, 2003). Eight poles of the $\mathrm{Nd}-\mathrm{Fe}-\mathrm{B}$ magnet are aligned on iron rods, and 12 rods are set on the back side of the microtiter plate to apply magnetic fields to the 96 wells. The magnetic field can be switched on (magnetic flux density: $318 \mathrm{mT}$ ) and off (magnetic flux density: $<10 \mathrm{mT}$ ) by rotating the rods $180^{\circ}$. The reaction station is combined with a heat block with a range of $4-99^{\circ} \mathrm{C}$ and is configured to perform the hybridization step. Heating and magnetic separation can be performed simultaneously in one well. This precise thermal control unit is suitable for DNA handling and has been used for DNA extraction, SNP detection in the genes for aldehyde dehydrogenase 2 (ALDH2) (Maruyama et al., 2004) and transforming growth factor (TGF) 
(Yoshino et al., 2010), detection of epidermal growth factor receptor (EGFR) mutations in non-small cell lung cancer (NSCLC), and determination of microsatellite repeats in the human thyroid peroxidase (TPOX) gene (Nakagawa et al., 2007).
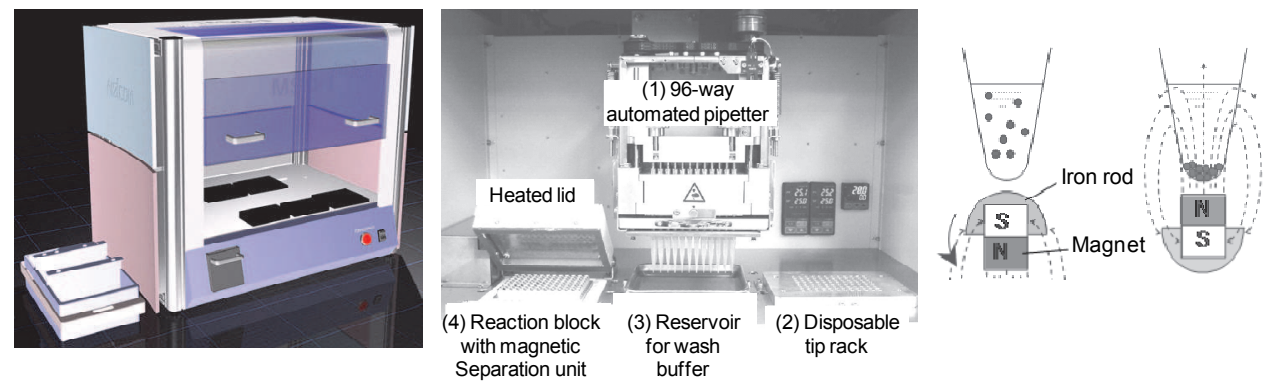

Fig. 9. Automated magnetic separation system, and magnetic separation is achieved in the bottom of microtiter plates.

Figure 10 shows the layout of an automated workstation with which magnetic particles can be separated on the inner surface of pipette tips. The automated system consists of an automated eight-way pipette bearing a retractable magnet mounted close to the pipette tips (1) a tip rack, (2) a reaction station for a 96-well microtiter plate, and (3) a luminescence detection unit. One rack can hold $8 \times 3$ tips for reactions. For automated magnetic separation, the suspension of magnetic particles is aspirated and dispersed using an automated pipette bearing a magnet. The automated pipette can move horizontally, and magnetic particles collected on the inner surface of pipette tips can be resuspended in the subsequent wells by the pipetting action (Matsunaga et al., 2007). As an advantage, this system can eliminate the carry-over of reaction mixtures to the following reaction steps. Due to precise liquid handling, this workstation is mainly used for highly-sensitive immunoassays, though its throughput capacity is less than the above system. Using this workstation, a fully-automated immunoassay was developed to detect EDCs (Matsunaga et al., 2003; Yoshino et al., 2008), human insulin (Tanaka and Matsunaga, 2000), and a prostate cancer marker (prostate specific antigen).
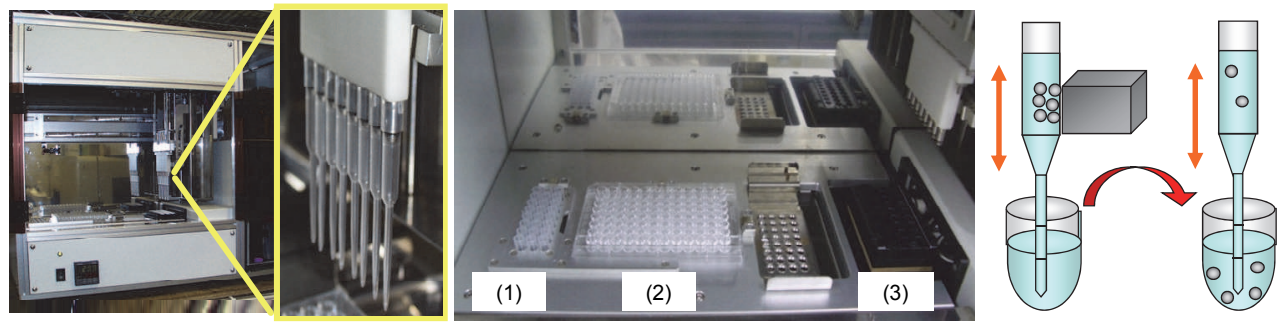

Fig. 10. Automated magnetic separation system, and magnetic separation is achieved on the inner surface of pipette tips.

Figure 11 shows the layout of an automated workstation with which magnetic particles can be collected onto a magnetic rod (Ota et al., 2006). This workstation is equipped with eight automated pestle units and a spectrophotometer that is interfaced with a photosensor 
amplifier. The magnetic rod that moves in both vertical and horizontal directions is composed of a neodymium-iron-boron (Nd-Fe-B) magnet pole and a covering sheath. DNA concentrations and purities are measured in the cuvette using an absorbance spectrometer that is integrated with the workstation. Light traverses the solution in the cuvette from bottom to top. When magnetic particles are collected from the reaction mixture, the core magnetic pole is sheathed, and then magnetic particles are suspended in the following step by stirring of the sheath without the core rod. The sheath is used as a pestle to gently mix the suspension of magnetic particles and solid samples. Using this system, DNA was directly extracted from dried maize powder using aminosilane-modified BacMPs. Furthermore, the quantitative detection of genetically-modified maize genes was examined by real-time PCR. This system offers rapid assay completion with high DNA yields and qualities comparable to those of conventional detergent-based methods.
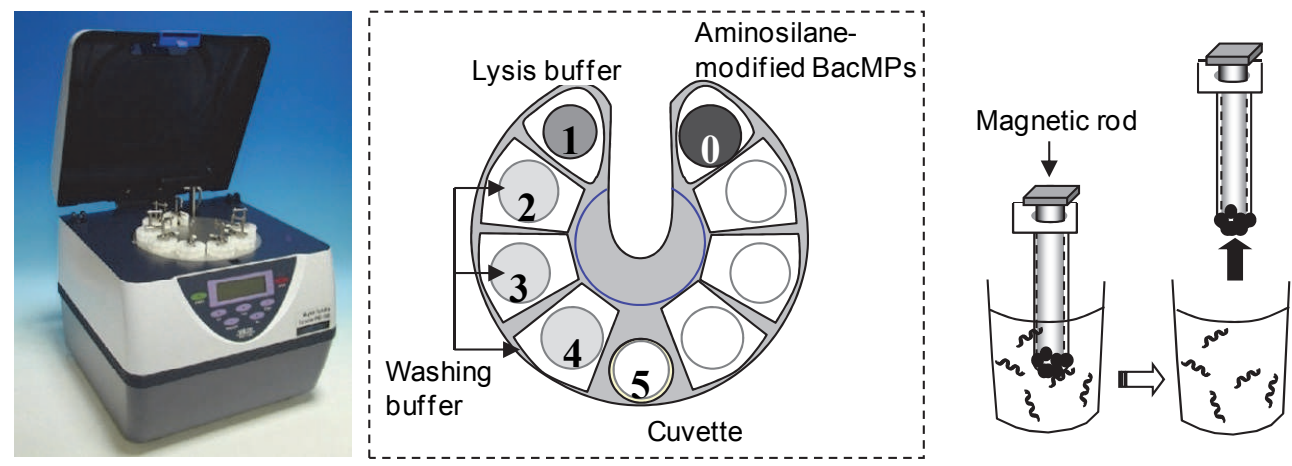

Fig. 11. Automated magnetic separation system, and magnetic separation is achieved onto a magnetic rod.

\section{Conclusion}

Magnetic particles have been utilized as biomolecule carriers since the 1970s when applied research examined bioreactors using enzyme-immobilized magnetic particles. Since then, various types of synthetic and bioengineered magnetic particles have been produced, modified, and enhanced. These magnetic particles have been widely used in place of centrifugation, filter, and chromatography separations, and applied to purifications of biological matter including target cells, proteins, and nucleic acids. More recent advances in the bioengineering of magnetic particles produced by magnetotactic bacteria have resulted in powerful tools for medical applications as well as basic research. This review focused on the applications of BacMPs for recovery or detections of bio-molecule. BacMPs are also available for other applications such as MRI contrast agents, and carriers for drug delivery systems, and so on. High potential magnetic particles will be developed combining with genetic engineering, and especially it is quite easy to control the kinds and numbers of proteins-displayed on BacMPs. Furthermore, it is possible to display artificially designed proteins or polypeptides onto BacMPs by the same methods. These approaches provide a new innovation in material science. Elucidation of the mechanism of magnetic particle formation in $M$. magneticum AMB-1 has provided a roadmap for designing novel biomaterials useful in multidisciplinary fields. 


\section{References}

Amemiya, Y., Arakaki, A., Staniland, S. S., Tanaka, T. \& Matsunaga, T. (2007). Controlled formation of magnetite crystal by partial oxidation of ferrous hydroxide in the presence of recombinant magnetotactic bacterial protein Mms6. Biomaterials 28: 5381-5389.

Arakaki, A., Webb, J. \& Matsunaga, T. (2003). A novel protein tightly bound to bacterial magnetic particles in Magnetospirillum magneticum strain AMB-1. Journal of Biological Chemistry 278(10): 8745-8750.

Baselt, D. R., Lee, G. U., Natesan, M., Metzger, S. W., Sheehan, P. E. \& Colton, R. J. (1998). A biosensor based on magnetoresistance technology. Biosensors $\mathcal{E}$ Bioelectronics 13(7-8): 731-739.

Blakemore, R. (1983). Magnetic bacteria and products derived therefrom. US4385119.

Deisenhofer, J. (1981). Crystallographic refinement and atomic models of a human Fc fragment and its complex with fragment B of protein A from Staphylococcus aureus at 2.9- and 2.8-.ANG. resolution. Biochemistry 20(9): 2361-2370.

Edelstein, R. L., Tamanaha, C. R., Sheehan, P. E., Miller, M. M., Baselt, D. R., Whitman, L. J. \& Colton, R. J. (2000). The BARC biosensor applied to the detection of biological warfare agents. Biosensors \& Bioelectronics 14(10-11): 805-813.

Eliasson, B. \& Kogelschatz, U. (1988). UV excimer radiation from dielectric-barrier discharges. Applied Physics B-Photophysics and Laser Chemistry 46(4): 299-303.

Fang, Y. L., Jayaram, H., Shane, T., Kolmakova-Partensky, L., Wu, F., Williams, C., Xiong, Y. \& Miller, C. (2009). Structure of a prokaryotic virtual proton pump at 3.2 angstrom resolution. Nature 460(7258): 1040-1043.

Gao, X. H., Cui, Y. Y., Levenson, R. M., Chung, L. W. K. \& Nie, S. M. (2004). In vivo cancer targeting and imaging with semiconductor quantum dots. Nature Biotechnology 22(8): 969-976.

Gleich, B. \& Weizenecker, R. (2005). Tomographic imaging using the nonlinear response of magnetic particles. Nature 435(7046): 1214-1217.

Goldman, E. R., Anderson, G. P., Tran, P. T., Mattoussi, H., Charles, P. T. \& Mauro, J. M. (2002). Conjugation of luminescent quantum dots with antibodies using an engineered adaptor protein to provide new reagents for fluoroimmunoassays. Analytical Chemistry 74(4): 841-847.

Gonzales, M. \& Krishnan, K. M. (2005). Synthesis of magnetoliposomes with monodisperse iron oxide nanocrystal cores for hyperthermia. Journal of Magnetism and Magnetic Materials 293(1): 265-270.

Graepler, F., Lauer, U. \& Gregor, M. (1998). Magnetic cell sorting for parietal cell purification using a new monoclonal antibody without influence on cell function. Journal of Biochemical and Biophysical Methods 36(2-3): 143-155.

Gref, R., Couvreur, P., Barratt, G. \& Mysiakine, E. (2003). Surface-engineered nanoparticles for multiple ligand coupling. Biomaterials 24(24): 4529-4537.

Gronenborn, A. M., Filpula, D. R., Essig, N. Z., Achari, A., Whitlow, M., Wingfield, P. T. \& Clore, G. M. (1991). A novel, highly stable fold of the immunoglobulin binding domain of streptococcal protein G. Science 253(5020): 657-661. 
Grubisha, D. S., Lipert, R. J., Park, H. Y., Driskell, J. \& Porter, M. D. (2003). Femtomolar detection of prostate-specific antigen: An immunoassay based on surfaceenhanced Raman scattering and immunogold labels. Analytical Chemistry 75(21): 5936-5943.

Gu, H. W., Ho, P. L., Tsang, K. W. T., Wang, L. \& Xu, B. (2003). Using biofunctional magnetic nanoparticles to capture vancomycin-resistant enterococci and other gram-positive bacteria at ultralow concentration. Journal of the American Chemical Society 125(51): 15702-15703.

Herr, J. K., Smith, J. E., Medley, C. D., Shangguan, D. \& Tan, W. (2006). Aptamerconjugated nanoparticles for selective collection and detection of cancer cells. Analytical Chemistry 78(9): 2918-2924.

Kettering, M., Winter, J., Zeisberger, M., Bremer-Streck, S., Oehring, H., Bergemann, C., Alexiou, C., Hergt, R., Halbhuber, K. J., Kaiser, W. A. \& Hilger, I. (2007). Magnetic nanoparticles as bimodal tools in magnetically induced labelling and magnetic heating of tumour cells: an in vitro study. Nanotechnology 18(17).

Kramer, R. M., Li, C., Carter, D. C., Stone, M. O. \& Naik, R. R. (2004). Engineered protein cages for nanomaterial synthesis. Journal of the American Chemical Society 126(41): 13282-13286.

Kuhara, M., Takeyama, H., Tanaka, T. \& Matsunaga, T. (2004). Magnetic cell separation using antibody binding with protein a expressed on bacterial magnetic particles. Analytical Chemistry 76(21): 6207-6213.

Lowenadler, B., Jansson, B., Paleus, S., Holmgren, E., Nilsson, B., Moks, T., Palm, G., Josephson, S., Philipson, L. \& Uhlen, M. (1987). A gene fusion system for generating antibodies against short peptides. Gene 58(1): 87-97.

Maruyama, K., Takeyama, H., Nemoto, E., Tanaka, T., Yoda, K. \& Matsunaga, T. (2004). Single nucleotide polymorphism detection in aldehyde dehydrogenase 2 (ALDH2) gene using bacterial magnetic particles based on dissociation curve analysis. Biotechnology and Bioengineering 87(6): 687-694.

Matsunaga, T. \& Kamiya, S. (1987). Use of magnetic particles isolated from magnetotactic bacteria for enzyme immobilization. Applied Microbiology and Biotechnology 26(4): 328-332.

Matsunaga, T., Maeda, Y., Yoshino, T., Takeyama, H., Takahashi, M., Ginya, H., Aasahina, J. \& Tajima, H. (2007). Fully automated immunoassay for detection of prostatespecific antigen using nano-magnetic beads and micro-poly styrene bead composites, 'Beads on Beads'. Analytica Chimica Acta 597(2): 331-339.

Matsunaga, T., Okamura, Y., Fukuda, Y., Wahyudi, A. T., Murase, Y. \& Takeyama, H. (2005). Complete genome sequence of the facultative anaerobic magnetotactic bacterium Magnetospirillum sp strain AMB-1. DNA Research 12(3): 157-166.

Matsunaga, T., Sato, R., Kamiya, S., Tanaka, T. \& Takeyama, H. (1999). Chemiluminescence enzyme immunoassay using ProteinA-bacterial magnetite complex. Journal of Magnetism and Magnetic Materials 194(1-3): 126-131.

Matsunaga, T., Takahashi, M., Yoshino, T., Kuhara, M. \& Takeyama, H. (2006). Magnetic separation of CD14(+) cells using antibody binding with protein A expressed on 
bacterial magnetic particles for generating dendritic cells. Biochemical and Biophysical Research Communications 350(4): 1019-1025.

Matsunaga, T., Togo, H., Kikuchi, T. \& Tanaka, T. (2000). Production of luciferasemagnetic particle complex by recombinant Magnetospirillum sp AMB-1. Biotechnology and Bioengineering 70(6): 704-709.

Matsunaga, T., Ueki, F., Obata, K., Tajima, H., Tanaka, T., Takeyama, H., Goda, Y. \& Fujimoto, S. (2003). Fully automated immunoassay system of endocrine disrupting chemicals using monoclonal antibodies chemically conjugated to bacterial magnetic particles. Analytica Chimica Acta 475(1-2): 75-83.

Matsunaga, T., Yoda, K., Udagawa, Y., Nemoto, E. \& Maruyama, K. (2003). Hybridization apparatus and method for detecting nucleic acid in sample using the same. US20030059823.

Miltenyi, S. (1995). Methods and materials for improved high gradient magnetic separation of biological materials. US5411863.

Miltenyi, S., Muller, W., Weichel, W. \& Radbruch, A. (1990). High gradient magnetic cell separation with MACS. Cytometry 11(2): 231-238.

Mirzabekov, T., Kontos, H., Farzan, M., Marasco, W. \& Sodroski, J. (2000). Paramagnetic proteoliposomes containing a pure, native, and oriented seven-transmembrane segment protein, CCR5. Nature Biotechnology 18(6): 649-654.

Mulder, W. J. M., Koole, R., Brandwijk, R. J., Storm, G., Chin, P. T. K., Strijkers, G. J., Donega, C. D., Nicolay, K. \& Griffioen, A. W. (2006). Quantum dots with a paramagnetic coating as a bimodal molecular imaging probe. Nano Letters 6(1): 16.

Nakagawa, T., Maruyama, K., Takeyama, H. \& Matsunaga, T. (2007). Determination of microsatellite repeats in the human thyroid peroxidase (TPOX) gene using an automated gene analysis system with nanoscale engineered biomagnetite. Biosensors \& Bioelectronics 22(9-10): 2276-2281.

Nakamura, C., Burgess, J. G., Sode, K. \& Matsunaga, T. (1995a). An iron-regulated gene, magA, encoding an iron transport protein of Magnetospirillum sp. strain AMB-1. Journal of Biological Chemistry 270(47): 28392-28396.

Nakamura, C., Kikuchi, T., Burgess, J. G. \& Matsunaga, T. (1995b). Iron-regulated expression and membrane localization of the magA protein in Magnetospirillum sp. strain AMB-1. Journal of Biochemistry 118(1): 23-27.

Nakamura, C., Sakaguchi, T., Kudo, S., Burgess, J. G., Sode, J. \& Matsunaga, T. (1993). Characterization of iron uptake in the magnetic bacterium Aquaspirillum sp. AMB-1. Applied Biochemistry and Biotechnology 37(39/40): 169-176.

Nakamura, M., Decker, K., Chosy, J., Comella, K., Melnik, K., Moore, L., Lasky, L. C., Zborowski, M. \& Chalmers, J. J. (2001). Separation of a breast cancer cell line from human blood using a quadrupole magnetic flow sorter. Biotechnology Progress 17(6): 1145-1155.

Ota, H., Lim, T. K., Tanaka, T., Yoshino, T., Harada, M. \& Matsunaga, T. (2006). Automated DNA extraction from genetically modified maize using aminosilanemodified bacterial magnetic particles. Journal of Biotechnology 125(3): 361-368. 
Pardoe, H., Clark, P. R., St Pierre, T. G., Moroz, P. \& Jones, S. K. (2003). A magnetic resonance imaging based method for measurement of tissue iron concentration in liver arterially embolized with ferrimagnetic particles designed for magnetic hyperthermia treatment of tumors. Magnetic Resonance Imaging 21(5): 483-488.

Patil, S., Sandberg, A., Heckert, E., Self, W. \& Seal, S. (2007). Protein adsorption and cellular uptake of cerium oxide nanoparticles as a function of zeta potential. Biomaterials 28: 4600-4607.

Perez, J. M., Josephson, L., O'Loughlin, T., Hogemann, D. \& Weissleder, R. (2002). Magnetic relaxation switches capable of sensing molecular interactions. Nature Biotechnology 20(8): 816-820.

Pileri, P., Uematsu, Y., Campagnoli, S., Galli, G., Falugi, F., Petracca, R., Weiner, A. J., Houghton, M., Rosa, D., Grandi, G. \& Abrignani, S. (1998). Binding of hepatitis C virus to CD81. Science 282(5390): 938-941.

Plank, C., Schillinger, U., Scherer, F., Bergemann, C., Remy, J. S., Krotz, F., Anton, M., Lausier, J. \& Rosenecker, J. (2003). The magnetofection method: Using magnetic force to enhance gene delivery. Biological Chemistry 384(5): 737-747.

Prasad, B. L. V., Stoeva, S. I., Sorensen, C. M., Zaikovski, V. \& Klabunde, K. J. (2003). Gold nanoparticles as catalysts for polymerization of alkylsilanes to siloxane nanowires, filaments, and tubes. Journal of the American Chemical Society 125(35): 10488-10489.

Sawakami-Kobayashi, K., Segawa, O., Obata, K., Hornes, E., Yohda, M., Tajima, H. \& Machida, M. (2003). Multipurpose robot for automated cycle sequencing. Biotechniques 34(3): 634-637.

Schotter, J., Kamp, P. B., Becker, A., Puhler, A., Reiss, G. \& Bruckl, H. (2004). Comparison of a prototype magnetoresistive biosensor to standard fluorescent DNA detection. Biosensors \& Bioelectronics 19(10): 1149-1156.

Schratzberger, P., Reinisch, N., Prodinger, W. M., Kahler, C. M., Sitte, B. A., Bellmann, R., FischerColbrie, R., Winkler, H. \& Wiedermann, C. J. (1997). Differential chemotactic activities of sensory neuropeptides for human peripheral blood mononuclear cells. Journal of Immunology 158(8): 3895-3901.

Schwalbe, M., Pachmann, K., Hoffken, K. \& Clement, J. H. (2006). Improvement of the separation of tumour cells from peripheral blood cells using magnetic nanoparticles. Journal of Physics-Condensed Matter 18(38): S2865-S2876.

Stampfli, M. R., Miescher, S., Aebischer, I., Zurcher, A. W. \& Stadler, B. M. (1994). Inhibition of human $\mathrm{IgE}$ synthesis by anti-IgE antibodies requires divalent recognition. European Journal of Immunology 24(9): 2161-2167.

Takahashi, M., Yoshino, T. \& Matsunaga, T. (2010). Surface modification of magnetic nanoparticles using asparagines-serine polypeptide designed to control interactions with cell surfaces. Biomaterials 31(18): 4952-4957.

Tanaka, T., Maruyama, K., Yoda, K., Nemoto, E., Udagawa, Y., Nakayama, H., Takeyama, H. \& Matsunaga, T. (2003). Development and evaluation of an automated workstation for single nucleotide polymorphism discrimination using bacterial magnetic particles. Biosensors \& Bioelectronics 19(4): 325-330. 
Tanaka, T. \& Matsunaga, T. (2000). Fully automated chemiluminescence immunoassay of insulin using antibody-protein A-bacterial magnetic particle complexes. Analytical Chemistry 72(15): 3518-3522.

Tanaka, T., Takeda, H., Kokuryu, Y. \& Matsunaga, T. (2004). Spontaneous integration of transmembrane peptides into a bacterial magnetic particle membrane and its application to display of useful proteins. Analytical Chemistry 76(13): 3764-3769.

Tiwari, A., Punshon, G., Kidane, A., Hamilton, G. \& Seifalian, A. M. (2003). Magnetic beads (Dynabead) toxicity to endothelial cells at high bead concentration: Implication for tissue engineering of vascular prosthesis. Cell Biology and Toxicology 19(5): 265-272.

Ugelstad, J., Berge, A., Ellingsen, T., Aune, O., Kilaas, L., Nilsen, T. N., Schmid, R., Stenstad, P., Funderud, S., Kvalheim, G., Nustad, K., Lea, T., Vartdal, F. \& Danielsen, H. (1988). Monosized magnetic particles and their use in selective cell separation. Makromolekulare Chemie-Macromolecular Symposia 17: 177-211.

Weissleder, R., Kelly, K., Sun, E. Y., Shtatland, T. \& Josephson, L. (2005). Cell-specific targeting of nanoparticles by multivalent attachment of small molecules. Nature Biotechnology 23: 1418-1423.

Wright, L. D., Cresson, E. L., Skeggs, H. R., Wood, T. R., Peck, R. L., Wolf, D. E. \& Folkers, K. (1952). Isolation of crystalline biocytin from yeast extract. Journal of the American Chemical Society 74(8): 1996-1999.

Xu, C. J., Xu, K. M., Gu, H. W., Zhong, X. F., Guo, Z. H., Zheng, R. K., Zhang, X. X. \& Xu, B. (2004). Nitrilotriacetic acid-modified magnetic nanoparticles as a general agent to bind histidine-tagged proteins. Journal of the American Chemical Society 126(11): 3392-3393.

Xu, L., Yu, H., Akhras, M. S., Han, S. J., Osterfeld, S., White, R. L., Pourmand, N. \& Wang, S. X. (2008). Giant magnetoresistive biochip for DNA detection and HPV genotyping. Biosensors \& Bioelectronics 24(1): 99-103.

Yoshino, T., Kaji, C., Nakai, M., Saito, F., Takeyama, H. \& Matsunaga, T. (2008). Novel method for evaluation of chemicals based on ligand-dependent recruitment of GFP labeled coactivator to estrogen receptor displayed on bacterial magnetic particles. Analytica Chimica Acta 626(1): 71-77.

Yoshino, T., Kato, F., Takeyama, H., Nakai, M., Yakabe, Y. \& Matsunaga, T. (2005). Development of a novel method for screening of estrogenic compounds using nano-sized bacterial magnetic particles displaying estrogen receptor. Analytica Chimica Acta 532(2): 105-111.

Yoshino, T. \& Matsunaga, T. (2005). Development of efficient expression system for protein display on bacterial magnetic particles. Biochemical and Biophysical Research Communications 338(4): 1678-1681.

Yoshino, T. \&Matsunaga, T. (2006). Efficient and stable display of functional proteins on bacterial magnetic particles using Mms13 as a novel anchor molecule. Applied and Environmental Microbiology 72(1): 465-471.

Yoshino, T., Nishimura, T., Mori, T., Suzuki, S., Kambara, H., Takeyama, H. \& Matsunaga, T. (2009). Nano-sized bacterial magnetic particles displaying pyruvate phosphate dikinase for pyrosequencing. Biotechnology and Bioengineering 103(1): 130-137. 
Yoshino, T., Shimojo, A., Maeda, Y. \& Matsunaga, T. (2010). Inducible Expression of Transmembrane Proteins on Bacterial Magnetic Particles in Magnetospirillum magneticum AMB-1. Applied and Environmental Microbiology 76(4): 1152-1157.

Yoshino, T., Takahashi, M., Takeyama, H., Okamura, Y., Kato, F. \& Matsunaga, T. (2004). Assembly of $G$ protein-coupled receptors onto nanosized bacterial magnetic particles using Mms16 as an anchor molecule. Applied and Environmental Microbiology 70(5): 2880-2885. 


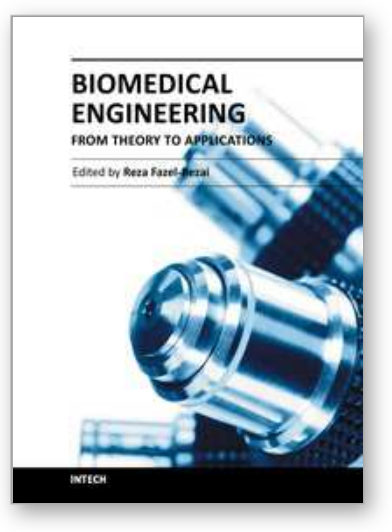

\author{
Biomedical Engineering - From Theory to Applications \\ Edited by Prof. Reza Fazel
}

ISBN 978-953-307-637-9

Hard cover, 486 pages

Publisher InTech

Published online 29, August, 2011

Published in print edition August, 2011

In all different areas in biomedical engineering, the ultimate objectives in research and education are to improve the quality life, reduce the impact of disease on the everyday life of individuals, and provide an appropriate infrastructure to promote and enhance the interaction of biomedical engineering researchers. This book is prepared in two volumes to introduce a recent advances in different areas of biomedical engineering such as biomaterials, cellular engineering, biomedical devices, nanotechnology, and biomechanics. It is hoped that both of the volumes will bring more awareness about the biomedical engineering field and help in completing or establishing new research areas in biomedical engineering.

\title{
How to reference
}

In order to correctly reference this scholarly work, feel free to copy and paste the following:

Tomoko Yoshino, Yuka Kanetsuki and Tadashi Matsunaga (2011). The Potential of Genetically Engineered Magnetic Particles in Biomedical Applications, Biomedical Engineering - From Theory to Applications, Prof. Reza Fazel (Ed.), ISBN: 978-953-307-637-9, InTech, Available from:

http://www.intechopen.com/books/biomedical-engineering-from-theory-to-applications/the-potential-ofgenetically-engineered-magnetic-particles-in-biomedical-applications

\section{INTECH}

open science | open minds

\section{InTech Europe}

University Campus STeP Ri

Slavka Krautzeka 83/A

51000 Rijeka, Croatia

Phone: +385 (51) 770447

Fax: +385 (51) 686166

www.intechopen.com

\section{InTech China}

Unit 405, Office Block, Hotel Equatorial Shanghai

No.65, Yan An Road (West), Shanghai, 200040, China

中国上海市延安西路65号上海国际贵都大饭店办公楼 405 单元

Phone: +86-21-62489820

Fax: +86-21-62489821 
(C) 2011 The Author(s). Licensee IntechOpen. This chapter is distributed under the terms of the Creative Commons Attribution-NonCommercialShareAlike-3.0 License, which permits use, distribution and reproduction for non-commercial purposes, provided the original is properly cited and derivative works building on this content are distributed under the same license. 